\title{
A New Fractional-Order Hyperchaotic System and Its Adaptive Tracking Control
}

\author{
Yinlan Chen, ${ }^{1}$ Haodong Zhang, ${ }^{2}$ and Xu Kong $\mathbb{D}^{3}$ \\ ${ }^{1}$ College of Mathematics and Statistics, Hubei Normal University, Huangshi 435002, China \\ ${ }^{2}$ Wuhan Research Institute of Posts and Telecommunications, Wuhan 430074, China \\ ${ }^{3}$ School of Mathematical Science, Liaocheng University, Liaocheng 252000, China \\ Correspondence should be addressed to Xu Kong; kongxu@lcu.edu.cn
}

Received 18 December 2020; Revised 30 January 2021; Accepted 5 February 2021; Published 15 February 2021

Academic Editor: Ailong Wu

Copyright ( $\odot 2021$ Yinlan Chen et al. This is an open access article distributed under the Creative Commons Attribution License, which permits unrestricted use, distribution, and reproduction in any medium, provided the original work is properly cited.

We construct for the first time a fractional-order hyperchaotic system via the original integer-order system. The dynamical behavior of this fractional-order hyperchaotic system is investigated in detail using first approximation method and Lyapunov exponents. Next, an adaptive control strategy for the univariate controlled hyperchaotic system has been proposed. Also, the tracking performance is fully taken into account in numerous applications, for instance, tracking sinusoidal periodic signal, selfsynchronization, and generalized synchronization of heterogeneous structure. Simulation results illustrate the validity and performability of the proposed adaptive tracking control scheme.

\section{Introduction}

Fractional calculus has the characteristics of historical dependence and global correlation and is an ideal tool to describe the memory and heredity of real problems. Compared with integer-order calculus, fractional calculus has better fitting degree with the experimental results in signal processing, hydrodynamics, mathematical biology, and electrochemistry [1-5]. Therefore, fractional calculus has been widely used in many disciplines and engineering fields. The research on fractional differential system can enrich the related results in the field of calculus, which has important theoretical significance and application value. Virtually, fractional calculus seems to be the direct extension of integral-order calculus, but the definition of fractionalorder derivative involves the kernel integral with parameter; then, many properties of integral-order differential system cannot hold in the fractional order, even if they have connotative relationships, these properties may not obtain equivalent conclusions [4]. Hence, it is of great significance to study the fractional differential system in depth. It is noted that qualitative problems of the nonlinear differential system (such as bifurcation, chaos, and quasiperiod) are an important branch of the evolution theory of differential equations. With the development of fractional calculus theory, the qualitative properties of fractional differential system are widely used in many fields, for example, physical mechanics, anomalous diffusion, automatic control, and biomedicine; the qualitative research of the fractional differential system has been paid attention by scholars, and many profound results have been obtained [1, 2, 4].

As a kind of motion form of the nonlinear system, chaos generally exists in nature. Chaos has many unique properties, such as aperiodic and long-term immeasurability. A chaotic system with two or more positive Lyapunov exponents is called the hyperchaotic system. Compared with chaos, the attractor orbits of the hyperchaotic system are separated in more directions [5-10]. As thus, the randomness and unpredictability about the hyperchaotic system are also stronger, which makes the hyperchaotic system have a broader development prospect in image encryption and secure communication. Based on the need of communication security, many scholars have tried to use hyperchaotic secure communication scheme, in which the key signal is generated by hyperchaotic system. Because of more complex dynamic characteristics in hyperchaotic 
secure communication scheme, it is difficult to use time series to reconstruct and decode the phase space. How to establish a hyperchaotic system model with new characteristics and how to control and synchronize hyperchaos have always been a hot topic in the field of dynamical systems and control $[8,10]$.

In the past few years, the control requirements of industrial system are improved rapidly. Accordingly, the nonlinear degree is increasing, which brings great challenges to the analysis and synthesis of the system. Therefore, it is very important for the stable operation of nonlinear system to improve the fault-tolerant ability and actively suppress external disturbances [3,11-15]. Adaptive control has the function of self-tuning, while tracking control has the function of path synchronization. The combination of adaptive control and tracking control can keep or approach the ideal performance of the original system after system failure [12-15]. The introduction of adaptive tracking control in the fractional-order system can not only upgrade the conventional adaptive tracking control but also meet the control requirements of the system described by the fractional-order operator. In the present stage, the research on fractional-order adaptive tracking control is still in its infancy, and only a basic theoretical framework has been formed. Therefore, it is necessary to further explore the potential of fractional-order adaptive tracking control in complex systems to achieve the asymptotic convergence of adaptive tracking control.

Based on the above analysis, in this paper, a new fourdimensional hyperchaotic system characterized by fractional differential equation is proposed, and dynamic behavior of the proposed system is studied. The stability of the equilibrium point and attractor evolution is studied by using the normal form method and Lyapunov exponents. The global dynamic behaviors, including hyperchaotic attractor, chaotic attractor, and singular degenerate ring, are discussed in detail. Moreover, adaptive tracking control of the considered system is analyzed and applied effectively.

\section{A New Fractional-Order Hyperchaotic System}

In memristor oscillator systems, a first-order mem-circuit is often discussed as follows:

$$
\left\{\begin{array}{l}
\dot{x}_{1}(t)=x_{2}(t) \\
\dot{x}_{2}(t)=\theta_{1} x_{3}(t)-\theta_{2} x_{2}(t)-\theta_{3} x_{1}^{2}(t) x_{2}(t) \\
\dot{x}_{3}(t)=\theta_{4} x_{2}(t)-\theta_{4} x_{3}(t)+\theta_{5} x_{4}(t) \\
\dot{x}_{4}(t)=-\theta_{6} x_{3}(t)-\theta_{7} x_{4}(t)
\end{array}\right.
$$

where $x_{i}(t), i \in\{1,2,3,4\}$, is the system state and $\theta_{i}$, $i \in\{1,2,3,4,5,6,7\}$, is the system parameter.

When $\theta_{1}=16.3, \theta_{2}=-3.28, \theta_{3}=19.68, \theta_{4}=1, \theta_{5}=1$, $\theta_{6}=15$, and $\theta_{7}=0.5$, system (1) shows a chaotic behavior. Accordingly, chaotic attractors in three-dimensional projection are described as Figure 1.

As a natural extension of integral calculus, fractional calculus plays an important role in practical engineering applications, especially in modeling the fractional-order system. Recently, Wu and Zeng [4] have introduced fractional calculus into the design of memristor systems, found that the memristor systems of fractional order have better performance, and have achieved some successful applications. Continuing along this path, we reestablish a new fractional-order system:

$$
\left\{\begin{array}{l}
D^{\alpha} x_{1}(t)=x_{2}(t) \\
D^{\alpha} x_{2}(t)=\theta_{1} x_{3}(t)-\theta_{2} x_{2}(t)-\theta_{3} x_{1}^{2}(t) x_{2}(t) \\
D^{\alpha} x_{3}(t)=\theta_{4} x_{2}(t)-\theta_{4} x_{3}(t)+\theta_{5} x_{4}(t) \\
D^{\alpha} x_{4}(t)=-\theta_{6} x_{3}(t)-\theta_{7} x_{4}(t)
\end{array}\right.
$$

where $D^{\alpha}$ is Caputo fractional derivative operator with order $\alpha>0, \quad x_{i}(t), \quad i \in\{1,2,3,4\}$, is the system state, and $\theta_{i}$, $i \in\{1,2,3,4,5,6,7\}$, is the system parameter.

When $\alpha=1$, system (2) degenerates into system (1). Compared with system (1), system (2) is a broader form.

Let $\alpha=0.5, \theta_{1}=16.3, \theta_{2}=-3.28, \theta_{3}=19.68, \theta_{4}=1$, $\theta_{5}=1, \theta_{6}=15$, and $\theta_{7}=0.5$; then, Lyapunov exponents of system (2) are $\lambda_{1}=0.400714, \quad \lambda_{2}=0.020389$, $\lambda_{3}=-0.008180$, and $\lambda_{4}=-7.854758$ (Figure 2); this suggests that system (2) is hyperchaotic. Accordingly, hyperchaotic attractors in three-dimensional projection are described as Figure 3.

\section{Dynamic Behaviors in Hyperchaotic System (2)}

By $\quad D^{\alpha} x_{1}(t)=0, \quad D^{\alpha} x_{2}(t)=0, \quad D^{\alpha} x_{3}(t)=0, \quad$ and $D^{\alpha} x_{4}(t)=0$, system (2) exhibits a unique equilibrium point $\mathrm{O}(0,0,0,0)$.

By linearizing system (2) at the equilibrium point $O(0,0,0,0)$, the corresponding Jacobian matrix is

$$
J=\left(\begin{array}{cccc}
0 & 1 & 0 & 0 \\
0 & -\theta_{2} & \theta_{1} & 0 \\
0 & \theta_{4} & -\theta_{4} & \theta_{5} \\
0 & 0 & -\theta_{6} & -\theta_{7}
\end{array}\right)
$$

The detailed characteristic equation is

$$
\begin{aligned}
\Upsilon(\chi)= & \chi\left[\chi^{3}+\left(\theta_{2}+\theta_{4}+\theta_{7}\right) \chi^{2}\right. \\
& +\left(-\theta_{1} \theta_{4}+\theta_{2} \theta_{4}+\theta_{2} \theta_{7}+\theta_{4} \theta_{7}+\theta_{5} \theta_{6}\right) \chi \\
& \left.-\theta_{1} \theta_{4} \theta_{7}+\theta_{2} \theta_{4} \theta_{7}+\theta_{2} \theta_{5} \theta_{6}\right] .
\end{aligned}
$$

Let $\alpha=0.5, \theta_{1}=16.3, \theta_{2}=-3.28, \theta_{3}=19.68, \theta_{4}=1$, $\theta_{5}=1, \theta_{6}=15$, and $\theta_{7}=0.5$ and the roots of the characteristic equation (4) are $\chi_{1}=0, \quad \chi_{2}=5.133$, $\chi_{3}=-1.676-2.946 i$, and $\chi_{4}=-1.676+2.946 i$, where $i$ is imaginary number; thus, the equilibrium point $O(0,0,0,0)$ is an unstable saddle point.

Suppose that the four Lyapunov exponents of system (2) are $\lambda_{1}, \lambda_{2}, \lambda_{3}$, and $\lambda_{4}$, by reordering to satisfy $\lambda_{1}>\lambda_{2}>\lambda_{3}>\lambda_{4}$; the following is to study the influences of parameter varying on the dynamic behavior of system (2); here, we select the evolution of Lyapunov exponents to reflect the influences of parameter variation. 


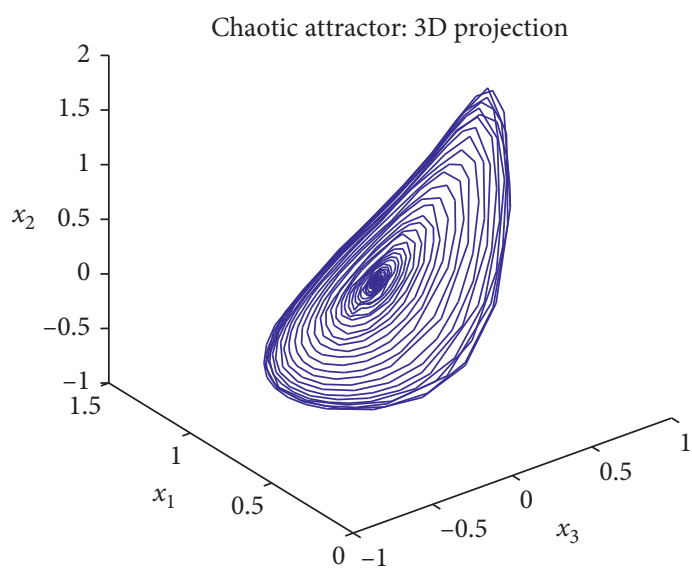

(a)

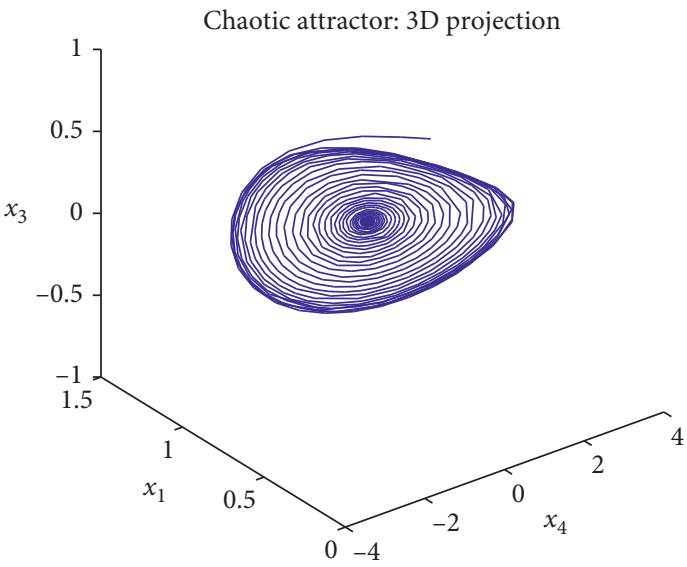

(c)

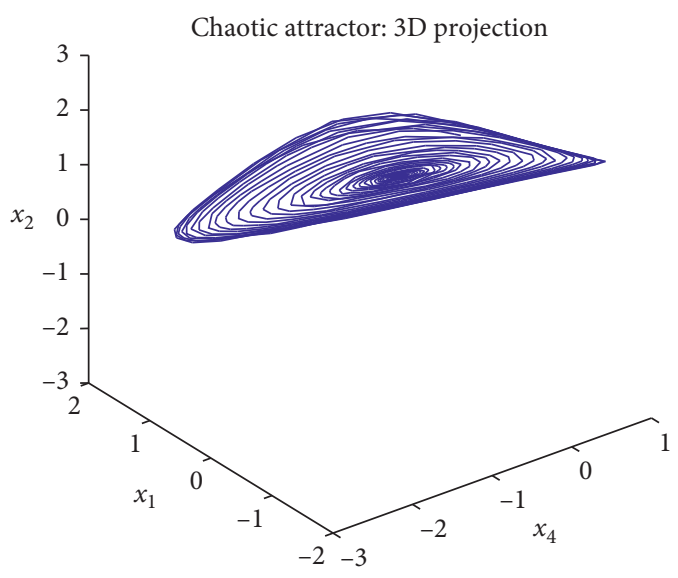

(b)

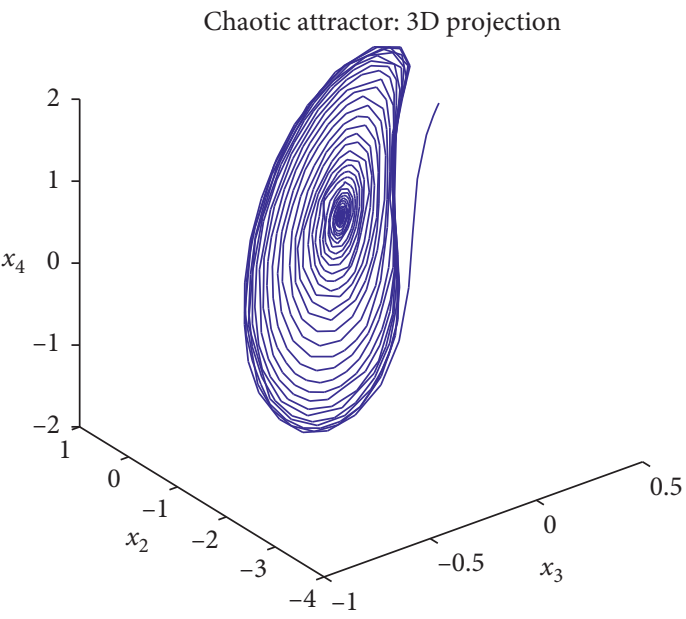

(d)

FIGURE 1: Chaotic attractors in three-dimensional projection.

Case 1: $\quad \alpha=0.5, \quad \theta_{1} \in[14.8,16.7], \quad \theta_{2}=-3.28$, $\theta_{3}=19.68, \theta_{4}=1, \theta_{5}=1, \theta_{6}=15$, and $\theta_{7}=0.5$; then, we get $\lambda_{1}>0, \lambda_{2}>0, \lambda_{3}<0$, and $\lambda_{4}<0$ (Figure 4 ); system (2) has two positive Lyapunov exponents and is in the hyperchaotic state.

Case 2: $\alpha=0.5, \theta_{1}=16.3, \theta_{2} \in[-4,-3], \theta_{3}=19.68$, $\theta_{4}=1, \theta_{5}=1, \theta_{6}=15$, and $\theta_{7}=0.5$; then, we get $\lambda_{1}>0$, $\lambda_{2}>0, \lambda_{3}<0$, and $\lambda_{4}<0$ (Figure 5); system (2) has two positive Lyapunov exponents and is in the hyperchaotic state.

Case 3: $\alpha=0.5, \theta_{1}=16.3, \theta_{2}=-3.28, \theta_{3} \in[19.4,20.6]$, $\theta_{4}=1, \theta_{5}=1, \theta_{6}=15$, and $\theta_{7}=0.5$; then, we get $\lambda_{1}>0$, $\lambda_{2}>0, \lambda_{3}<0$, and $\lambda_{4}<0$ (Figure 6); system (2) has two positive Lyapunov exponents and is in the hyperchaotic state.

Case 4: $\alpha=0.5, \theta_{1}=16.3, \theta_{2}=-3.28, \theta_{3}=19.68$, $\theta_{4} \in[0.8,9], \theta_{5}=1, \theta_{6}=15$, and $\theta_{7}=0.5$; then, we get $\lambda_{1}>0, \lambda_{2}>0, \lambda_{3}<0$, and $\lambda_{4}<0$; system (2) has two positive Lyapunov exponents and is in the hyperchaotic state.

Case 5: $\alpha=0.5, \theta_{1}=16.3, \theta_{2}=-3.28, \theta_{3}=19.68$, $\theta_{4}=1, \theta_{5} \in[0.2,1.4], \theta_{6}=15$, and $\theta_{7}=0.5$; then, we

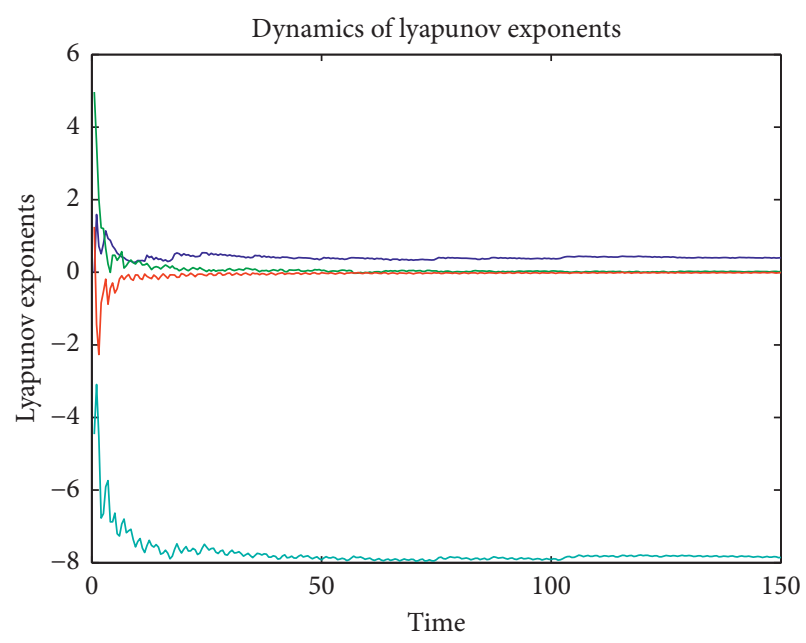

FIGURe 2: Dynamics of Lyapunov exponents with respect to system (2).

get $\lambda_{1}>0, \lambda_{2}>0, \lambda_{3}<0$, and $\lambda_{4}<0$; system (2) has two positive Lyapunov exponents and is in the hyperchaotic state. 


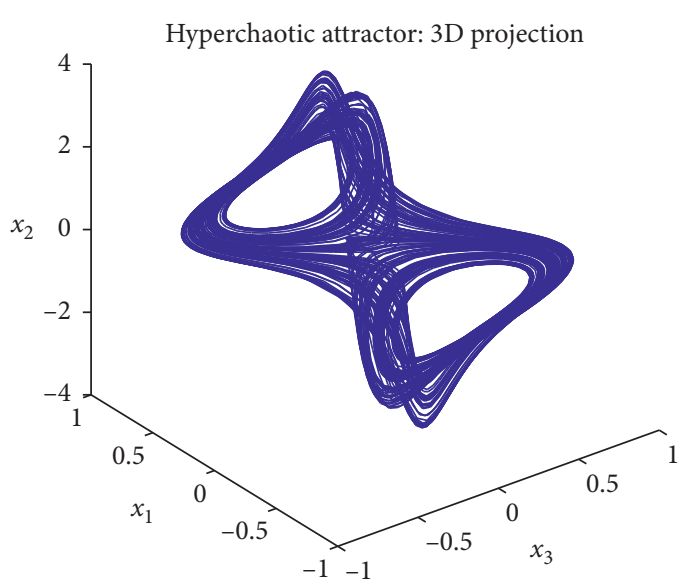

(a)

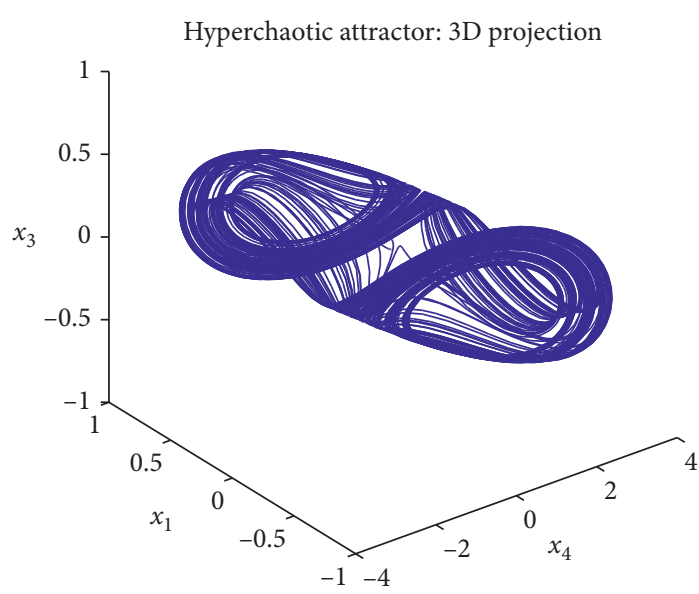

(c)

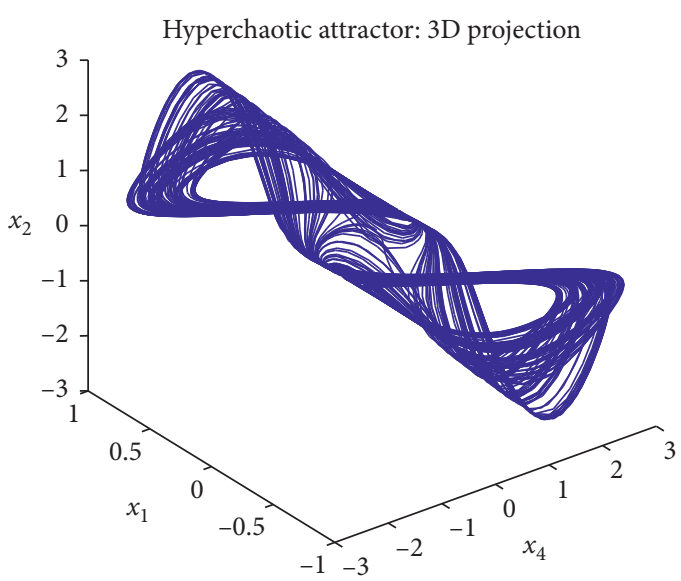

(b)

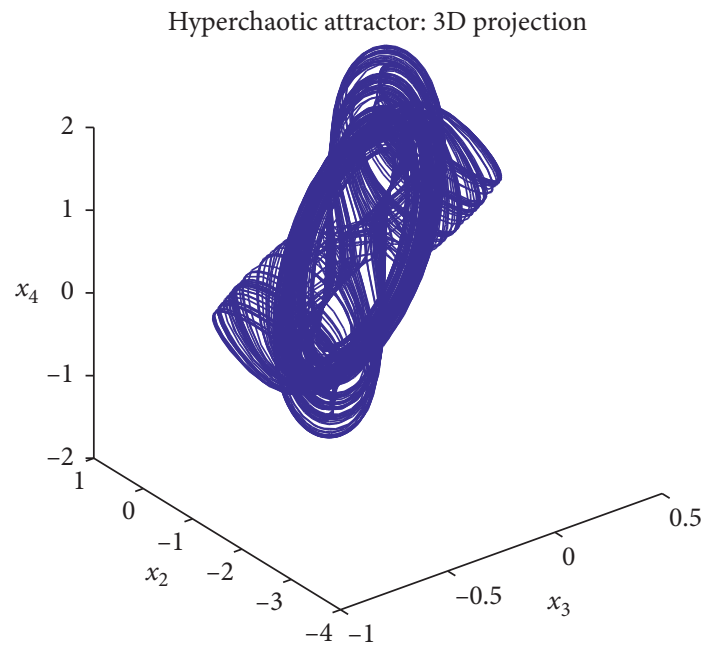

(d)

FIGURE 3: Hyperchaotic attractors in the three-dimensional projection.

Case 6: $\alpha=0.5, \theta_{1}=16.3, \theta_{2}=-3.28, \theta_{3}=19.68$, $\theta_{4}=1, \theta_{5}=1, \theta_{6} \in[14,21.3]$, and $\theta_{7}=0.5$; then, we get $\lambda_{1}>0, \lambda_{2}>0, \lambda_{3}<0$, and $\lambda_{4}<0$; system (2) has two positive Lyapunov exponents and is in the hyperchaotic state.

Case 7: $\alpha=0.5, \theta_{1}=16.3, \theta_{2}=-3.28, \theta_{3}=19.68$, $\theta_{4}=1, \theta_{5}=1, \theta_{6}=15$, and $\theta_{7} \in[0,0.5]$; then, we get $\lambda_{1}>0$ and $\lambda_{2}>0$ (for example, $\theta_{7}=0,0.1,0.2,0.3,0.5$ ) or $\lambda_{2}<0$ (for example, $\theta_{7}=0.4$ ), $\lambda_{3}<0, \lambda_{4}<0$; system (2) will experience hyperchaotic and chaotic states; see Figure 7 when $\theta_{7}=0.2$ and Figure 8 when $\theta_{7}=0.4$.

The algorithm for Lyapunov exponents is described as follows:

Step 1: the fractional differential equation is transformed into an equivalent integral equation

Step 2: the approximate solution of the transformed equation system is obtained

Step 3: the Jacobian matrix of the transformed equation system is decomposed via $Q R$ method (the matrix is

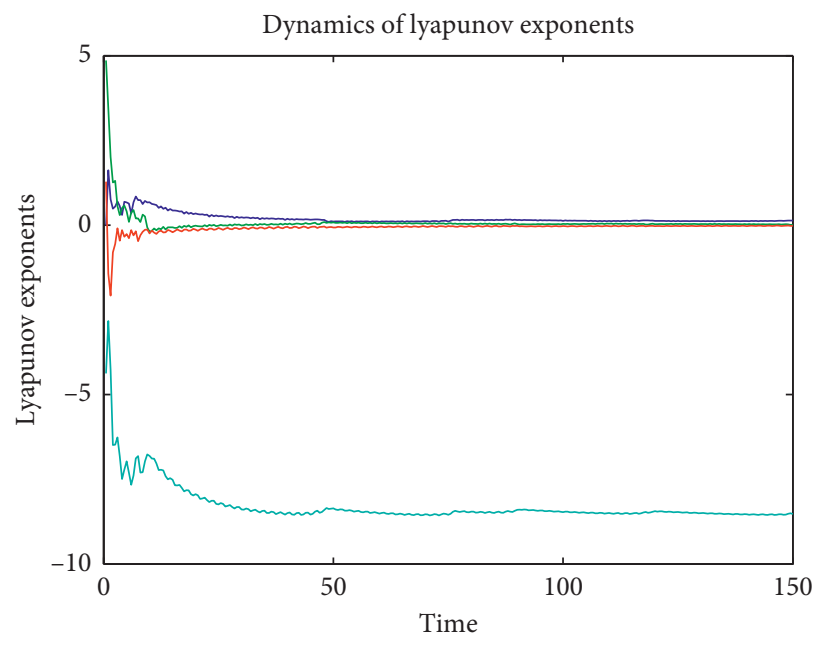

FIgURe 4: Dynamics of Lyapunov exponents with respect to Case 1.

decomposed into a normal orthogonal matrix $Q$ and an upper triangular matrix $R$ ) 


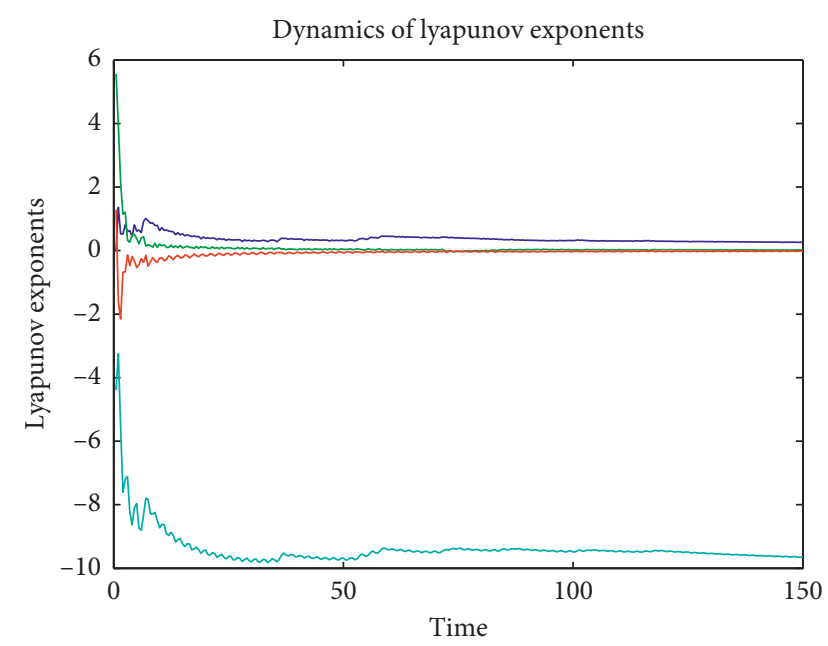

FIGURe 5: Dynamics of Lyapunov exponents with respect to Case 2.

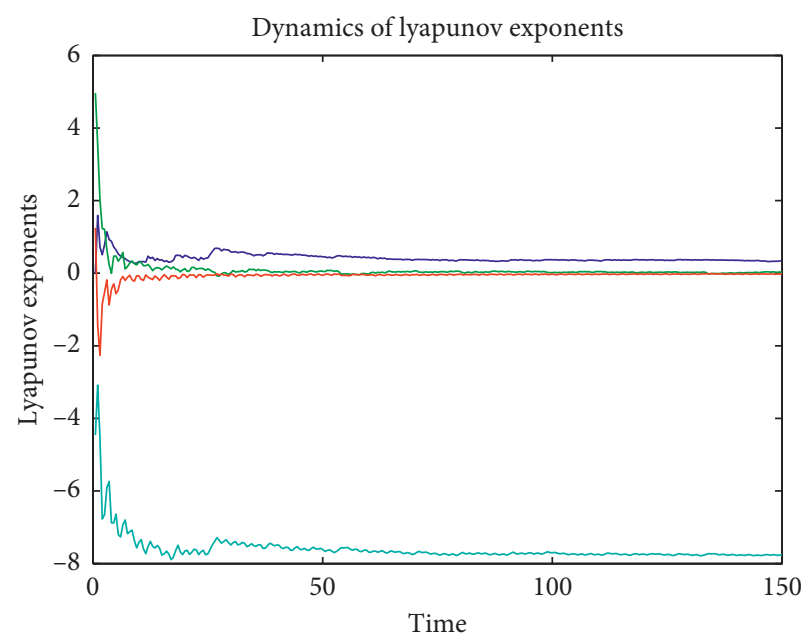

Figure 6: Dynamics of Lyapunov exponents with respect to Case 3.

Step 4: the product of eigenvalues of Jacobian matrix is calculated

Step 5: Lyapunov exponents and fractional dimension are calculated

Remark 1. Lyapunov exponents represent the numerical characteristics of the average divergence rate of adjacent trajectories in phase space, which is one of the characteristics used to identify some numerical values of chaotic motion. Here, we transform the fractional differential equation into an equivalent integral equation and solve the Lyapunov exponents of the corresponding integral equation via MATLAB.

Remark 2. In Cases 1-6, by adjusting the parameters, we describe the hyperchaotic behavior of the system. At the same time, in order to provide an intuitive understanding, we give the evolution diagram of Lyapunov exponents for Case 1 to Case 3 (of course, the similar evolution diagram of Lyapunov exponents for Case 4 to Case 6 is omitted). In Case
7, when $\theta_{7} \in[0,0.5]$, the system will be hyperchaotic/ chaotic.

\section{Adaptive Tracking Control}

4.1. Design Strategy about Adaptive Tracking Control. Combined with the nonlinear characteristics of system (2), this subsection aims to carry out the research on adaptive tracking control strategy of the fractional-order hyperchaotic system. Our goal is to design an adaptive tracking controller so that the variable $x_{1}(t)$ of the hyperchaotic system (2) can track any given reference signal in various forms.

Consider the following univariate controlled hyperchaotic system:

$$
\left\{\begin{array}{l}
D^{\alpha} x_{1}(t)=x_{2}(t)+u(t) \\
D^{\alpha} x_{2}(t)=\theta_{1} x_{3}(t)-\theta_{2} x_{2}(t)-\theta_{3} x_{1}^{2}(t) x_{2}(t) \\
D^{\alpha} x_{3}(t)=\theta_{4} x_{2}(t)-\theta_{4} x_{3}(t)+\theta_{5} x_{4}(t) \\
D^{\alpha} x_{4}(t)=-\theta_{6} x_{3}(t)-\theta_{7} x_{4}(t)
\end{array}\right.
$$

where $u(t)$ is the adaptive controller to be designed.

Suppose any reference signal is $y(t)$, define the tracking error as $e(t)=x_{1}(t)-y(t)$ and the controller $u(t)$ is designed as

$$
u(t)=\rho(t) e(t)
$$

where $\rho(t)$ is adaptive control gain and satisfies

$$
D^{\alpha} \rho^{2}(t)=-2 \omega\left[e^{2}(t)+e(t) D^{\alpha} e(t)\right],
$$

where $\omega>0$ is a constant and its size of $\omega$ directly affects the convergence rate of control gain $\rho(t)$.

Theorem 1. For the controlled system (5), if the adaptive controller represented by (6) and (7) is selected, then the state variable $x_{1}(t)$ can track any given reference signal $y(t)$ in different forms.

Proof. To choose

$$
V(t)=\frac{1}{2} e^{2}(t)+\frac{1}{2 \omega} \rho^{2}(t)
$$

then

$$
\begin{aligned}
D^{\alpha} V(t) & \leq e(t) D^{\alpha} e(t)+\frac{1}{\omega} \rho(t) D^{\alpha} \rho(t) \\
& =-e^{2}(t) \\
& \leq 0 .
\end{aligned}
$$

By mean of the fractional-order convergence principle, it follows that the state variable $x_{1}(t)$ of the controlled system (5) asymptotically converging to the reference signal $y(t)$ under the adaptive controllers (6) and (7).

4.2. Tracking Sinusoidal Periodic Signal. Signal tracking is an important research topic in the field of signal processing. It is widely used in radar detection, sonar positioning, wireless 


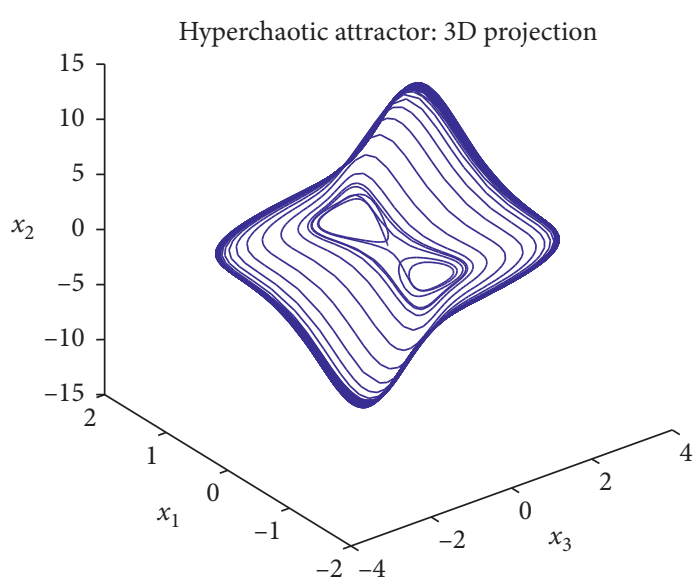

(a)

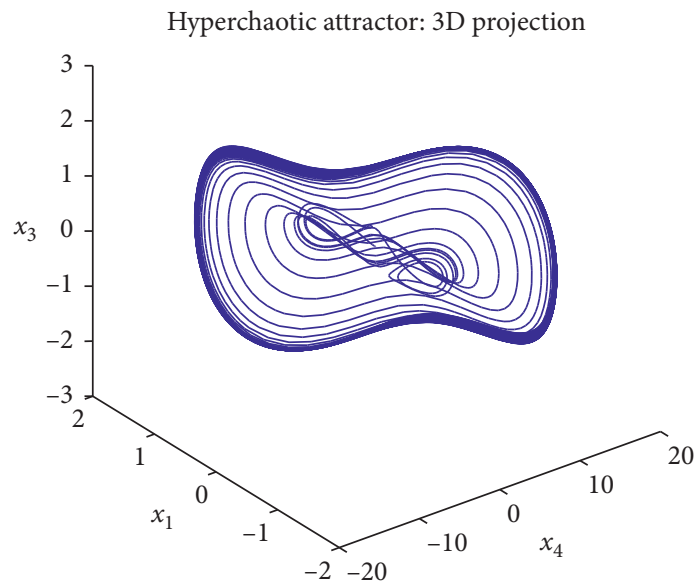

(c)

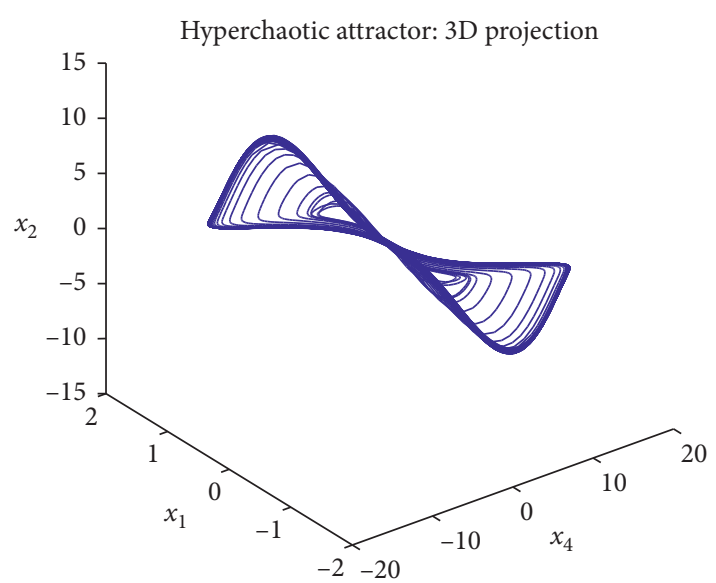

(b)

Hyperchaotic attractor: 3D projection

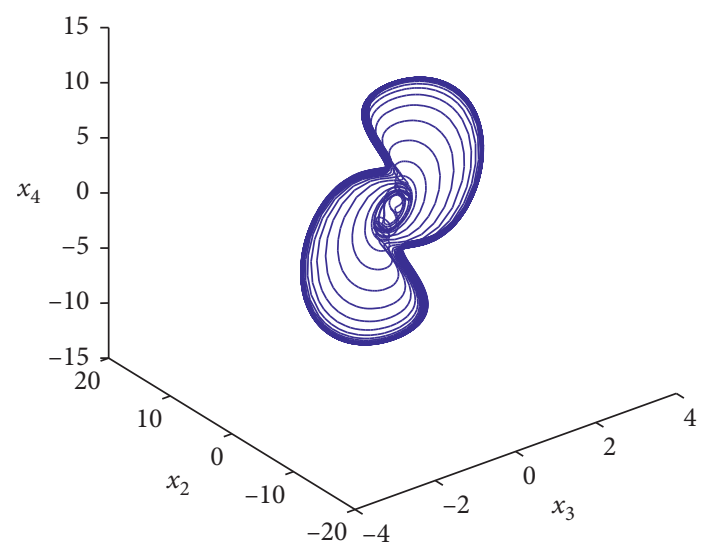

(d)

Figure 7: Hyperchaotic attractors.

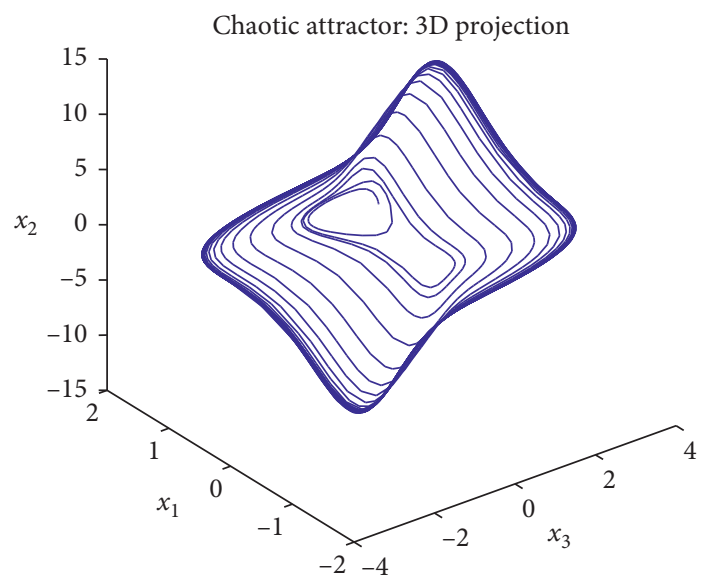

(a)

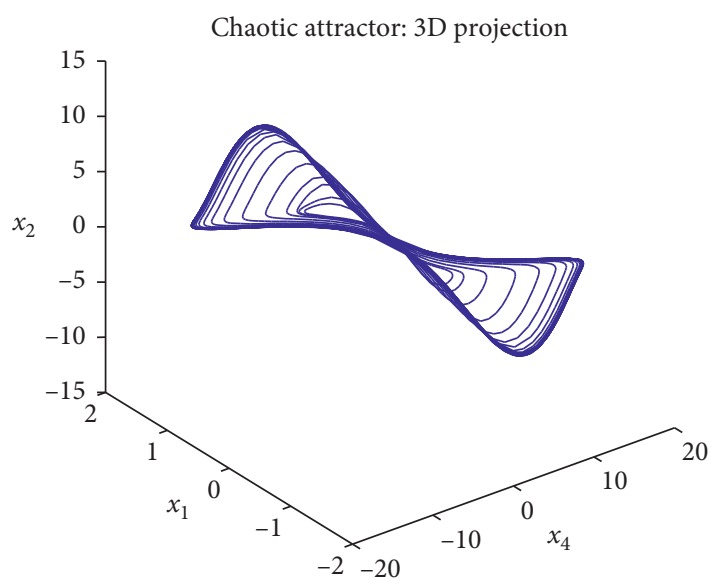

(b)

FIgURE 8: Continued. 


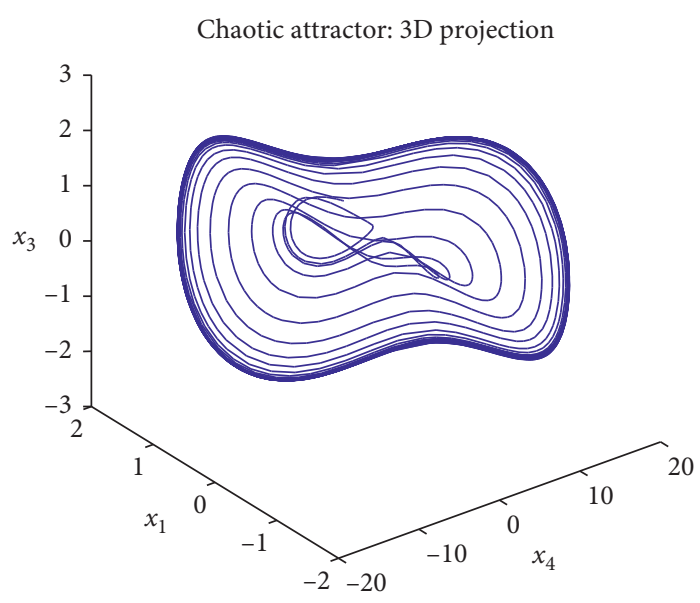

(c)

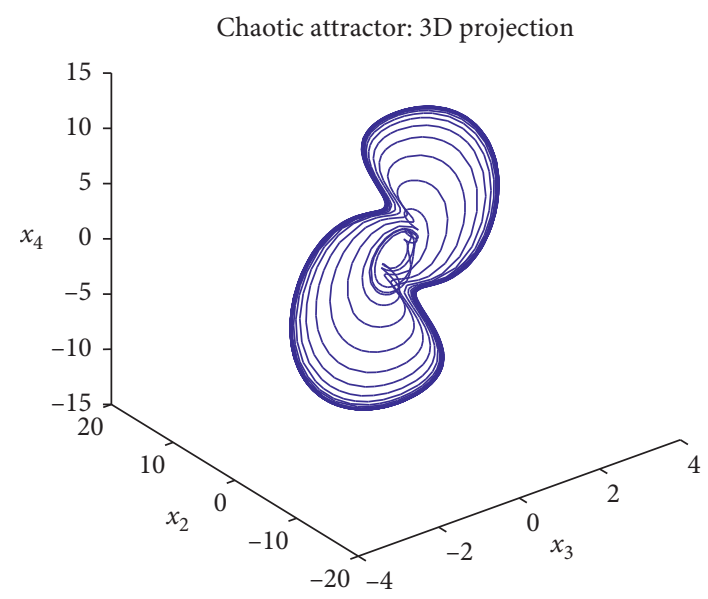

(d)

Figure 8: Chaotic attractors.

communication, seismic exploration, radio astronomy, biomedical engineering, and many other military and civil fields. Signal tracking performance includes noise performance (noise filtering ability), dynamic performance (dynamic tracking ability), and tracking performance (tracking accuracy). The adaptive tracking control strategy proposed in this paper can satisfy the dynamic signal tracking ability without losing the tracking performance, so as to improve the loop performance.

Here, the sinusoidal periodic signal $0.5 \sin (t)$ is selected as the reference signal, and the simulation result is shown in Figure 9. It can be seen from Figure 9 that the system variable $x_{1}(t)$ is controlled on the periodic orbit after about 0.1 second, which fully demonstrates that the designed adaptive tracking controller can make the controlled system (5) accurately track the given periodic signal $0.5 \sin (t)$.

4.3. Self-Synchronization. The phenomenon of self-synchronization opens up a new application domain of vibration technology and guides the birth of a new discipline of vibration utilization engineering. At present, self-synchronous vibrator is widely used in industrial production departments, such as vibrating screen, vibrating feeder, and vibrating dehydrator. On the basis of the analysis of adaptive tracking control in the previous content, combining the adaptive tracking control with the self-synchronization theory, we aim to guide the establishment of a systematic self-synchronization theoretical framework.

Selecting the following hyperchaotic system (10) as the response system,

$$
\left\{\begin{array}{l}
D^{\alpha} z_{1}(t)=z_{2}(t) \\
D^{\alpha} z_{2}(t)=\theta_{1} z_{3}(t)-\theta_{2} z_{2}(t)-\theta_{3} z_{1}^{2}(t) z_{2}(t) \\
D^{\alpha} z_{3}(t)=\theta_{4} z_{2}(t)-\theta_{4} z_{3}(t)+\theta_{5} z_{4}(t) \\
D^{\alpha} z_{4}(t)=-\theta_{6} z_{3}(t)-\theta_{7} z_{4}(t)
\end{array}\right.
$$

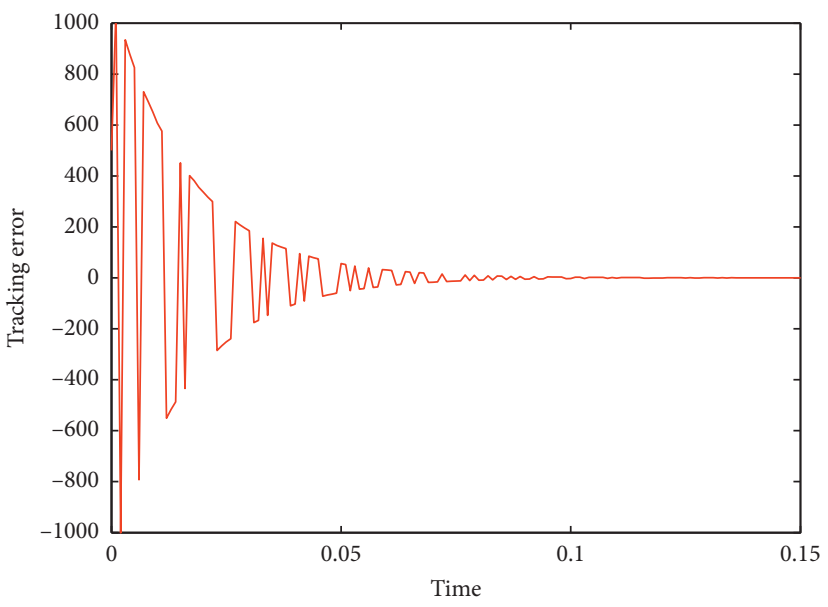

FIGURE 9: The curve of tracking error.

Let $\alpha=0.5, \theta_{1}=16.3, \theta_{2}=-3.28, \theta_{3}=19.68, \theta_{4}=1$, $\theta_{5}=1, \theta_{6}=15$, and $\theta_{7}=0.5$; to choose the reference signal $y(t)=z_{1}(t)$, the relevant simulation result is shown as Figure 10. Viewed from Figure 10, system state $x_{1}(t)$ is consistent with $z_{1}(t)$ about 0.2 second, which also completely demonstrates that system (5) and system (10) can achieve self-synchronization under the adaptive controllers (6) and (7).

\subsection{Generalized Synchronization of Heterogeneous Structure.} Heterogeneous coupling is one of the key characteristics of the cyber-physical system. In fact, the communication network in the cyber-physical system is composed of sensor network, actuator network, and computer network, which is often heterogeneous. How to choose the appropriate and effective control strategy to obtain desired performance of the system and then to achieve the stability and controllability of the system are hot issues. 


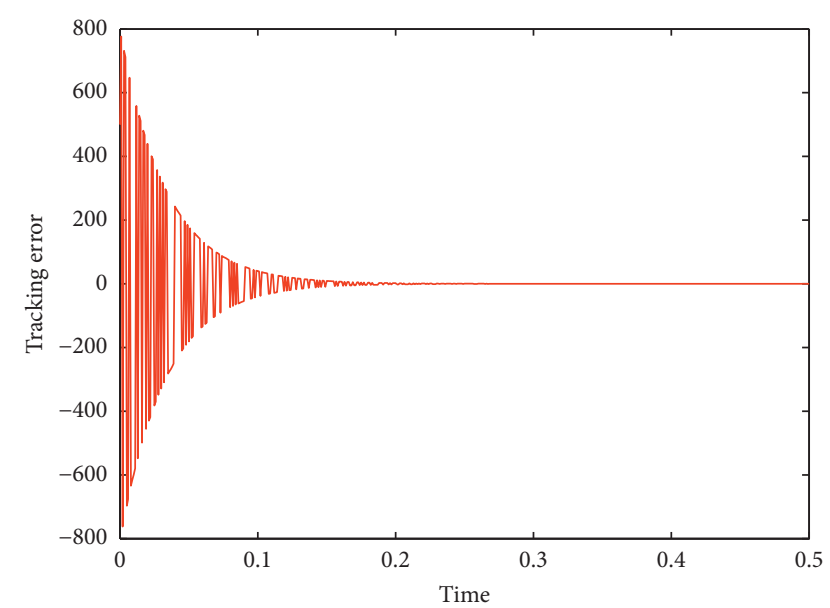

Figure 10: The curve of tracking error.

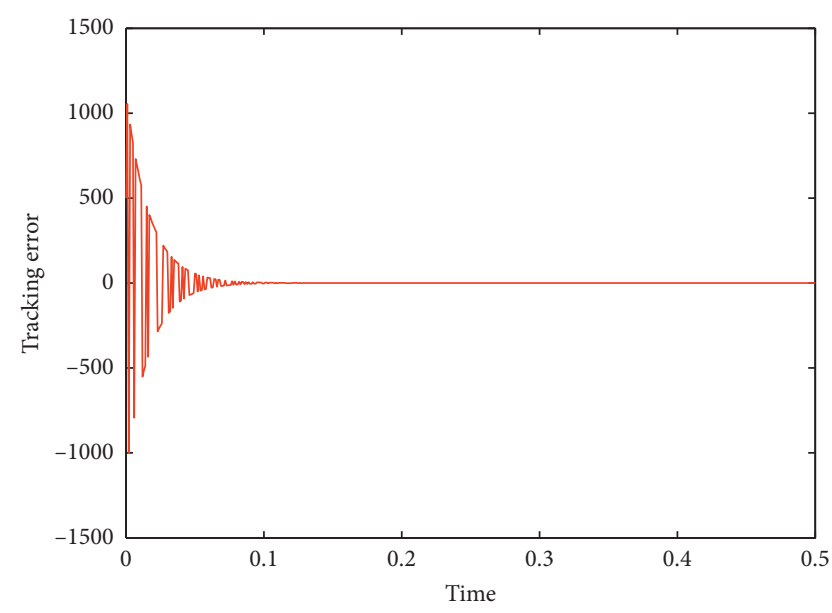

Figure 11: The curve of tracking error.

Selecting the following three-dimensional system (11) as the response system,

$$
\left\{\begin{array}{l}
D^{\alpha} \mu_{1}(t)=\xi_{1}\left(\mu_{2}(t)-\mu_{1}(t)\right) \\
D^{\alpha} \mu_{2}(t)=\xi_{2} \mu_{1}(t)-\xi_{3} \mu_{1}(t) \mu_{3}(t) \\
D^{\alpha} \mu_{3}(t)=-\xi_{4} \mu_{3}(t)+\xi_{5} \mu_{1}^{2}(t)
\end{array}\right.
$$

When $\alpha=0.5, \xi_{1}=10, \xi_{2}=40, \xi_{3}=1, \xi_{4}=2.5$, and $\xi_{5}=4$, system (11) shows chaotic behavior. Assume the reference signal $y(t)=\mu_{1}(t)+\mu_{2}(t)$; in other words, generalized synchronization is realized in system (5) and system (11). The related simulation result is shown as Figure 11. As seen from Figure 11, system state $x_{1}(t)$ is consistent with the reference signal $y(t)=\mu_{1}(t)+\mu_{2}(t)$ about 0.1 second, which also fully illustrates that system (5) and system (11) in heterogeneous structure can achieve generalized synchronization under the adaptive controllers (6) and (7).

\section{Conclusion}

In this paper, based on an integer-order chaotic system, a new fractional-order hyperchaotic system is constructed.
The basic dynamics of this system, including Lyapunov exponents and attractor types, are studied in detail. The analysis shows that the new fractional-order system presents chaos, hyperchaos, and other complex motions with the change of different parameters. Finally, an adaptive tracking controller is designed to realize the single-variable tracking control of the hyperchaotic system for different types of reference signals. Taking the tracking control of sinusoidal periodic signal, self-synchronization, and generalized synchronization between heterogeneous systems as examples, the effectiveness of the adaptive controller is verified.

\section{Data Availability}

No data were used to support this study.

\section{Conflicts of Interest}

The authors declare that there are no conflicts of interest regarding the publication of this paper.

\section{References}

[1] K. Mathiyalagan and G. Sangeetha, "Second-order sliding mode control for nonlinear fractional-order systems," Applied Mathematics and Computation, vol. 383, Article ID 125264, 2020.

[2] E. Saatci and E. Saatci, "State-space analysis of fractionalorder respiratory system models," Biomedical Signal Processing and Control, vol. 57, Article ID 101820, 2020.

[3] P. Trivedi, V. Vyawahare, and M. D. Patil, "Tracking control for fractional order systems with high relative degree outputs," IFAC-PapersOnLine, vol. 53, no. 1, pp. 170-175, 2020.

[4] A. Wu and Z. Zeng, "Global Mittag-Leffler stabilization of fractional-order memristive neural networks," IEEE Transactions on Neural Networks and Learning Systems, vol. 28, no. 1, pp. 206-217, 2017.

[5] A. Yousefpour, H. Jahanshahi, J. M. Munoz-Pacheco, S. Bekirosd, and Z. Wei, "A fractional-order hyper-chaotic economic system with transient chaos, Chaos," Solitons \& Fractals, vol. 130, Article ID 109400, 2020.

[6] V. Basios and C. G. Antonopoulos, "Hyperchaos \& labyrinth chaos: revisiting Thomas-Rössler systems," Journal of Theoretical Biology, vol. 460, pp. 153-159, 2019.

[7] F. F. Franco, E. L. Rempel, and P. R. Munoz, "Crisis and hyperchaos in a simplified model of magnetoconvection," Physica D, vol. 406, Article ID 132417, 2020.

[8] A. E. Matouk, "Stability conditions, hyperchaos and control in a novel fractional order hyperchaotic system," Physics Letters $A$, vol. 373, no. 25, pp. 2166-2173, 2009.

[9] M. Patra and S. Banerjee, "Hyperchaos in 3-D piecewise smooth maps, Chaos," Solitons \& Fractals, vol. 133, Article ID 109681, 2020.

[10] F. G. Prants and P. C. Rech, "Suppression of periodic structures and the onset of hyperchaos in a parameter-space of the Baier-Sahle flow," Chaos, Solitons \& Fractals, vol. 83, pp. 105-111, 2016.

[11] A. Wu, H. Liu, and Z. Zeng, "Observer design and $\mathrm{H} \infty$ performance for discrete-time uncertain fuzzy-logic systems," IEEE Transactions on Cybernetics, p. 1. In press, 2020.

[12] N. Koksal, H. An, and B. Fidan, "Backstepping-based adaptive control of a quadrotor UAV with guaranteed tracking performance," ISA Transactions, vol. 105, pp. 98-110, 2020. 
[13] M. Labbadi and M. Cherkaoui, "Robust adaptive nonsingular fast terminal sliding-mode tracking control for an uncertain quadrotor UAV subjected to disturbances," ISA Transactions, vol. 99, pp. 290-304, 2020.

[14] J. Lee, D.-E. Kang, and C. Park, "Geometric robust adaptive control for satellite attitude tracking with reaction wheels," Acta Astronautica, vol. 179, pp. 238-252, 2021.

[15] A. Palacio-Betancur and M. G. Soto, "Adaptive tracking control for real-time hybrid simulation of structures subjected to seismic loading," Mechanical Systems and Signal Processing, vol. 134, Article ID 106345, 2019. 